Document downloaded from:

http://hdl.handle.net/10251/82509

This paper must be cited as:

Rodríguez Egea, PL.; Lozano Juste, J. (2015). Unnatural agrochemical ligands for engineered abscisic acid receptors. Trends in Plant Science. 20(6):330-332. doi:10.1016/j.tplants.2015.04.001.

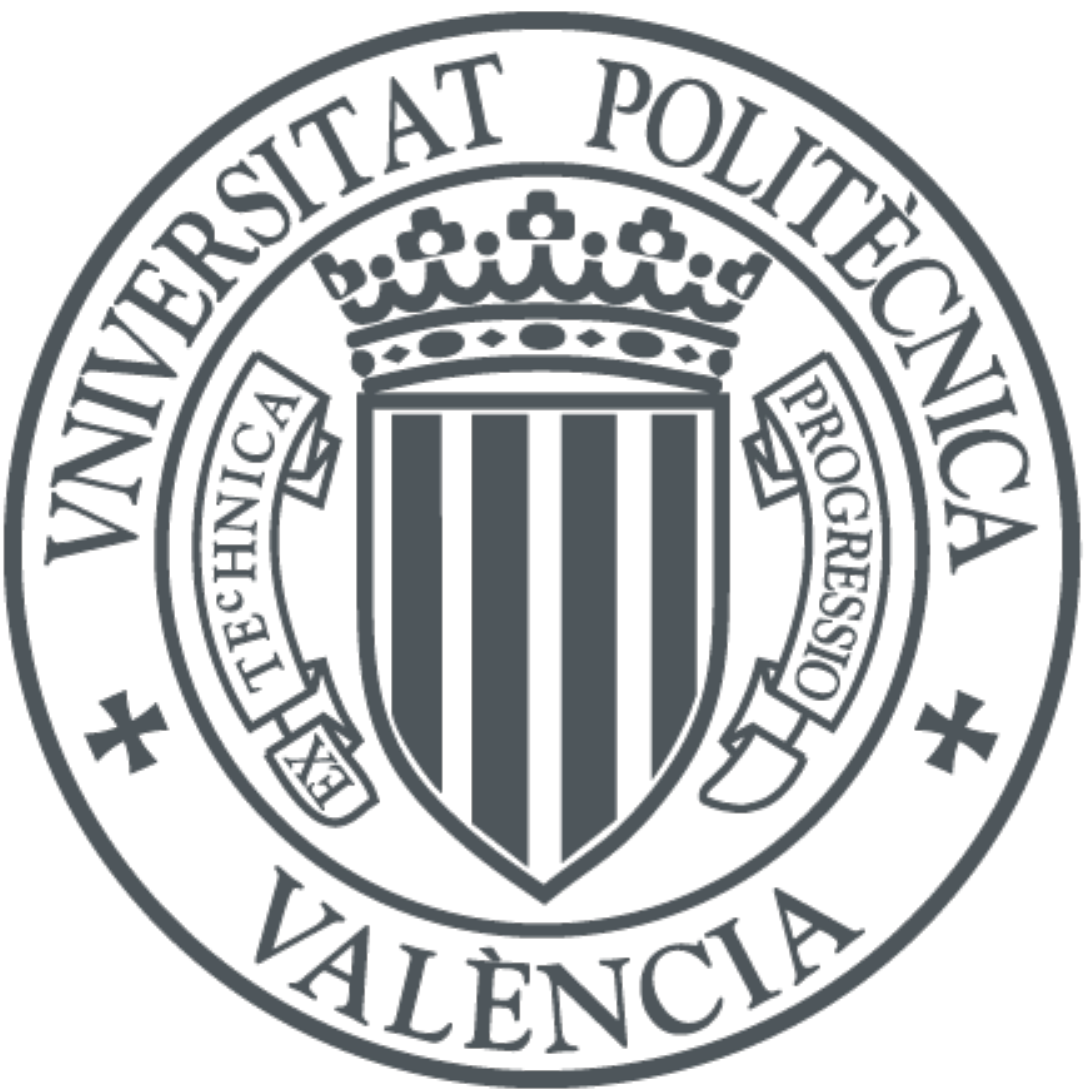

The final publication is available at

http://doi.org/10.1016/j.tplants.2015.04.001

Copyright Elsevier (Cell Press)

Additional Information 


\section{Unnatural agrochemical ligands for engineered abscisic acid 2 receptors}

3

$4 \quad$ Pedro L. Rodriguez ${ }^{1}$ and Jorge Lozano-Juste ${ }^{2}$

$5{ }^{1}$ Instituto de Biología Molecular y Celular de Plantas, Consejo Superior de

6 Investigaciones Científicas-Universidad Politécnica de Valencia, 46022

7 Valencia, Spain

$8{ }^{2}$ Department of Botany and Plant Sciences. Center for Plant Cell Biology.

9 Institute for Integrative Genome Biology. University of California, Riverside, CA, 10 92521

12 Corresponding author: Rodriguez, P.L. (prodriguez@ibmcp.upv.es)

Abstract

17 Existing agrochemicals can be endowed with new applications through protein engineering of plant receptors. A recent study shows an engineered PYR1 ABA receptor can be activated by mandipropamid. Plants engineered with such PYR1 variant are responsive to this agrochemical, which confers protection against drought through activation of ABA signaling.

Keywords: agrochemical, engineered ABA receptor, unnatural ligand, orthogonal ligand-receptor. 
Plant growth is severely impaired by adverse environmental conditions such as drought, salinity, cold or high temperature, which can reduce average productivity of crops by $50-80 \%$. In particular, the limited water availability is the single most important factor that reduces crop yield and $70 \%$ of fresh water is currently used by agriculture globally [1]. Therefore, engineering plants with enhanced drought tolerance is expected to have a major impact leading to more sustainable cropping systems worldwide. Scientists sometimes claim that drought stress is as complicated to plant biology as cancer is to mammalian biology. However, decades of research focused to elucidate the molecular mechanisms that govern plant adaptive responses to drought have been fruitful in providing molecular insights into the problem. Moreover, it has been demonstrated that plants are amenable to improved stress tolerance through several mechanisms of action. For instance, it is widely recognized that the phytohormone abscisic acid (ABA) represents a crucial signal for plant response to drought. ABA plays a pivotal role to mount and coordinate the drought adaptive response since ABA signaling leads to both regulation of plant water use and adaptive transcriptional responses to avoid or resist drought conditions.

A major breakthrough in ABA perception and signaling occurred in 2009 with the discovery of the PYR/PYL/RCAR family of ABA receptors and the in vitro reconstitution of the pathway. ABA elicits plant responses through binding to soluble PYRABACTIN RESISTANCE1 (PYR1)/PYR1-LIKE (PYL)/REGULATORY COMPONENTS OF ABA RECEPTORS (RCAR) receptors, which constitute a multigene family. PYR/PYL/RCAR receptors perceive $A B A$ intracellularly and as a result, form ternary complexes with clade A protein phosphatases type $2 \mathrm{C}$ (PP2Cs), thereby inactivating them [2,3]. This prevents the PP2C-mediated dephosphorylation of ABA-activated sucrose nonfermenting 1-related protein kinases ( $\mathrm{SnRKs}$ ) subfamily 2 (SnRK2s), which results in the activation of a SnRK2-dependent phosphorylation cascade affecting a high number of targets in the plant cell [4,5]. Activation of ABA signaling in this ligand-dependent manner leads to plant protection during water deficit (Figure 1). Future applications in agriculture were envisaged when the 
structural and mechanistic insights into ABA signaling were unveiled. For instance, the recent development of small synthetic molecules acting as ABAagonists (bind to the $A B A$ receptor and activate $A B A$ signaling) or $A B A$ antagonists (occupy the receptor and block ABA-mediated responses) offers key tools to modulate ABA signaling and potential use in agriculture [6-8] (Figure 1). Spraying with ABA-agonists could turn on the ABA stress response pathway, which regulates both water loss and root growth, promotes accumulation of compatible solutes and synthesis of protective dehydrins. ABAantagonists could favor seed germination or might prevent overactivation of ABA signaling in pollen [8]. However, these chemicals have yet to be approved for safe use in agriculture. Alternatively, constitutive or inducible overexpression of improved ABA receptors in transgenic crops might also represent a valuable strategy that does not require the addition of exogenous compounds $[9,10]$.

Sir Frances Bacon published in 1625: "if the hill will not come to you, you must go to the hill". Thus, if current agrochemicals do not positively influence plant response to drought yet, the scientific community must endow them with such new property. In this direction, Park et al., [11] report a new pioneering strategy to trigger the plant $A B A$ response using engineered $A B A$ receptors that are able to accommodate already-in use agrochemicals. These authors started their work with 15 agrochemicals that lack the capability to act as ligands of $A B A$ receptors. Obviously, these chemicals were not designed to this end because $A B A$ receptors show high specificity for their natural ligand. However, after saturation mutagenesis of 25-ABA ligand proximal residues of the PYR1 ABA receptor [9], the authors were able to identify engineered PYR1 receptors that bind weakly to 4 of the 15 compounds tested (Figure 1). Additional rounds involving combinatorial, saturation and shuffling mutagenesis allowed the generation of a hextuple PYR1 mutant, named PYR $1^{\text {MANDI }}$, which possesses nanomolar sensitivity for one agrochemical named mandipropamid (MD) to inhibit in vitro the PP2C HYPERSENSITIVE TO ABA (HAB1) (Figure 1). This compound is currently used to control oomycete pathogens, formerly known as pseudo-fungi or water mold, but currently belonging to a different supergroup of the domain Eukarya than Fungi. Among the oomycetes, there are severe plant pathogens, e.g. Plasmopara viticola and Phytophthora infestans. This latter 
oomycete caused the great potato blight and famine in Ireland (1845-1850). $P$. infestans is still a serious disease and it is controlled by regular spraying with agrochemicals, such as MD. Since the PYR $1^{\text {MANDI }}$ receptor is now able to accommodate the agrochemical, which was also documented to atomic resolution by elucidating $\mathrm{X}$-ray crystal structure of a ternary complex with the mutated receptor and $\mathrm{HAB1}$, a new use has been established for MD, i.e. the activation of $A B A$ signaling in transgenic plants expressing PYR1 ${ }^{\text {MANDI }}$. To this end the authors were able to show that MD induces an ABA-like transcriptional response and is able to control transpiration and drought tolerance in

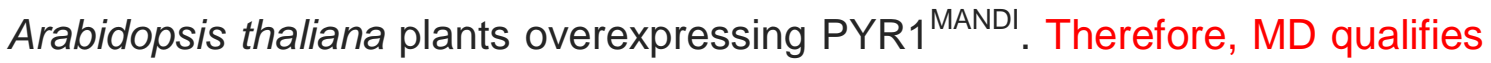
as orthogonal agonist of the ABA pathway since it is silent (with respect to ABA signaling) in normal cells but in transgenic cells expressing the neo-receptor PYR $1^{\text {MANDI }}$ triggers activation of ABA signaling.

In tomato (Solanum lycopersicum) plants expressing the 35S:PYR1 ${ }^{\text {MANDI }}$ transgene, the combination of MD+PYR $1^{\text {MANDI }}$ was able to control leaf temperature, suggesting that this approach might be useful to modulate $A B A$ signaling in crops. A potential limitation of this strategy would be that transgenic PYR/PYL ABA receptors were not able to efficiently inhibit crop PP2Cs, since higher specificity for the receptor-phosphatase interaction might be expected when homologous components interact each other. However, it has been shown that tomato PYR/PYL ABA receptors are able to inhibit Arabidopsis PP2Cs and confer enhanced drought tolerance when overexpressed in Arabidopsis [12]. Thus, it seems that $A B A$ receptors can be functionally exchanged between species. Additionally, crop ABA receptors could be tailored to efficiently bind agrochemicals. Although further experiments are needed in different crop species, it might be expected that this proof of the concept could be efficiently translated into transgenic crops to enhance drought tolerance. Thus, if this dream becomes reality, it could happen that an agrochemical able to prevent severe oomycete-promoted plant diseases gains the ability to confer plant drought tolerance in crops. In the absence of the engineered ABA receptor, the agrochemical only would exert its original function, which avoids undesirable side effects when treating oomycete infections. 
127 Work in author laboratory was supported by the Ministerio de Ciencia e Innovacion, Fondo Europeo de Desarrollo Regional and Consejo Superior de Investigaciones Cientificas (grant BIO2011-23446). Authors acknowledge to Sevier Medical Art for sharing images.

Figure 1. ABA signaling is controlled in a ligand-dependent manner. (a) Dimeric PYR1, a member of the PYR/PYL/RCAR family of ABA receptors, perceives ABA intracellularly and in its monomeric state forms stable ternary complexes with clade A PP2Cs, thereby inactivating them. It allows the phosphorylation and activation of SnRK2s, which in turn phosphorylate different targets in plant cells to mount the ABA response under stress conditions. (b) Different approaches have been followed to identify small molecules able to act as agonists or antagonists of ABA signaling. The structure of a 3'-alkylsulfanyl-ABA with a 6-C extension (AS6), which acts as ABA-antagonist, is shown. Quinabactin is a sulfonamide ABA-agonist. Mandipropamid is a mandelamide orthogonal agonist able to activate an engineered PYR1 receptor (PYR1 ${ }^{\text {MANDI}}{ }^{\text {). }}$ Images were generated with the LigandScout software tool using 3NEF, 3QN1, 4LA7 and 4WVO Protein Data Bank codes. (c) An agrochemical can be converted into an ABA orthogonal agonist through the use of engineered $A B A$ receptors. First, the K59R mutation abolishes ABA-binding capacity of PYR1. Second, weak binding of agrochemicals can be detected in receptor allele collections where the ABA-binding pocket has been mutagenized. Finally, combinatorial and shuffling mutagenesis generates an engineered PYR1 receptor that shows high affinity for binding of a certain agrochemical. (d) The ABA signaling pathway can be activated in Arabidopsis or tomato plants expressing PYR1 ${ }^{\text {MANDI }}$ through mandipropamid treatment. 


\section{References}

1 Pennisi, E. (2008). Plant genetics. The blue revolution, drop by drop, gene by gene. Science 320, 171-173.

2 Ma, Y. et al. (2009). Regulators of PP2C Phosphatase Activity Function as Abscisic Acid Sensors. Science 324, 1064-1068

3 Park, S.Y. et al. (2009). Abscisic Acid Inhibits Type 2C Protein Phosphatases via the PYR/PYL Family of START Proteins. Science 324, 1068-1071.

4 Umezawa, T. et al. (2013). Genetics and phosphoproteomics reveal a protein phosphorylation network in the abscisic acid signaling pathway in Arabidopsis thaliana. Sci. Signal. 6, rs8

5 Wang, P. et al. (2013). Quantitative phosphoproteomics identifies SnRK2 protein kinase substrates and reveals the effectors of abscisic acid action. Proc. Natl. Acad. Sci. U. S. A 110, 11205-11210.

6 Okamoto, M. et al. (2013). Activation of dimeric ABA receptors elicits guard cell closure, ABA-regulated gene expression, and drought tolerance. Proc. Natl. Acad. Sci. U. S. A 110, 12132-12137.

7 Cao, M. et al. (2013). An ABA-mimicking ligand that reduces water loss and promotes drought resistance in plants. Cell Res. 23, 1043-1054.

8 Takeuchi, J. et al. (2014). Designed abscisic acid analogs as antagonists of PYL-PP2C receptor interactions. Nat. Chem. Biol. 10, 477-482.

9 Mosquna, A. et al., (2011). Potent and selective activation of abscisic acid receptors in vivo by mutational stabilization of their agonist-bound conformation. Proc. Natl. Acad. Sci. U. S. A 108, 20838-20843.

10 Pizzio, G.A et al., (2013). The PYL4 A194T mutant uncovers a key role of PYR1-LIKE4/PROTEIN PHOSPHATASE 2CA interaction for abscisic acid signaling and plant drought resistance. Plant Physiol 163, 441-455.

11 Park, S.Y. et al. (2015). Agrochemical control of plant water use using engineered abscisic acid receptors. Nature doi: 10.1038/nature1412.

12 Gonzalez-Guzman, M. et al. (2014). Tomato PYR/PYL/RCAR abscisic acid receptors show high expression in root, differential sensitivity to the abscisic acid agonist quinabactin, and the capability to enhance plant drought resistance. J. Exp. Bot. 65, 4451-4464. 
To the attention of the referees,

We acknowledge very much the different comments to improve the ms. Corrections to address all referee's suggestions are labeled in red. Additionally, we have introduced some changes concerning oomycetes. Upon discussion with experts in taxonomy, we realized that oomycetes (formerly known as pseudo-fungi or water mold) currently belong to a different supergroup of the domain Eukarya than Fungi. Therefore, although the agrochemical is still sold as "fungicide", it is not correct to match oomycetes and fungi.

Concerning the comment of reviewer 2, we note that in the absence of the engineered $A B A$ receptor, mandipropamid only would exert its original function, which avoids undesirable side effects when treating oomycete infections in non-transgenic plants. If necessary, other agrochemicals would be available to treat oomycete infection in transgenic plants expressing PYR1 ${ }^{\text {MANDI }}$.

Sincerely

Dr Pedro L. Rodriguez Egea

Investigador científico del CSIC

Instituto de Biologia Molecular y Celular de Plantas

Consejo Superior de Investigaciones Científicas-Univ. Politécnica

Avd de los Naranjos. Edificio CPI, 8E

ES-46022 Valencia.Spain

Phone $\quad 34963877860$

Fax $\quad 34963877859$

E-mail prodriguez@ibmcp.upv.es 
(a)
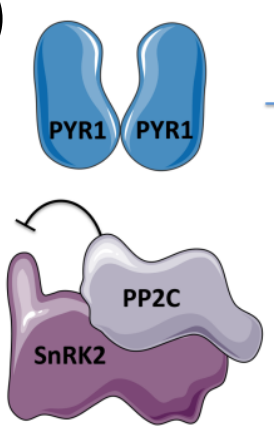

$\downarrow$

ABA signaling OFF
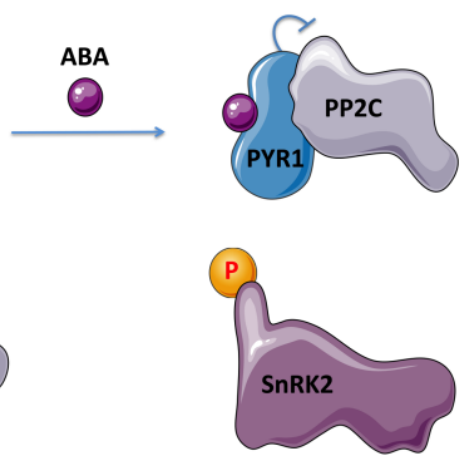

$\downarrow$

ABA signaling ON

(b)

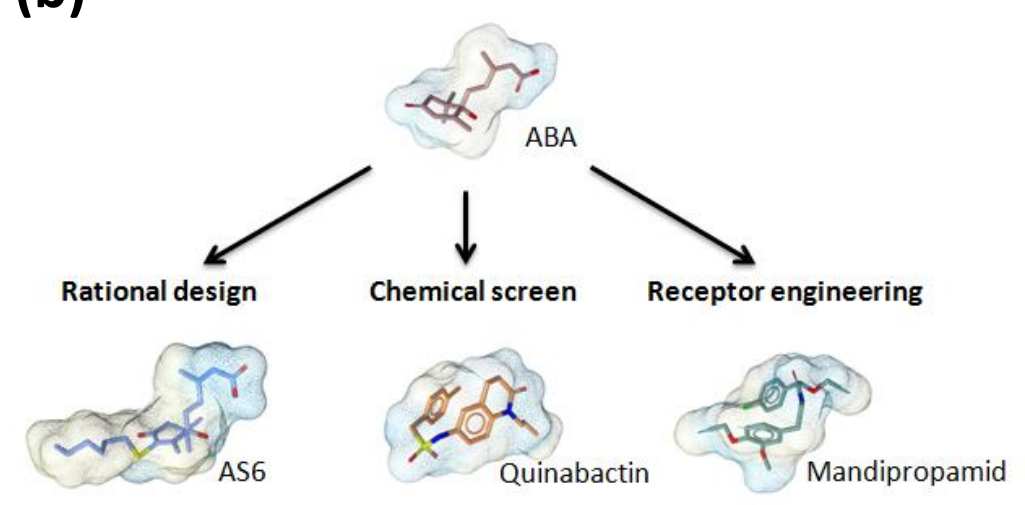

Antagonist
Agonist (c)

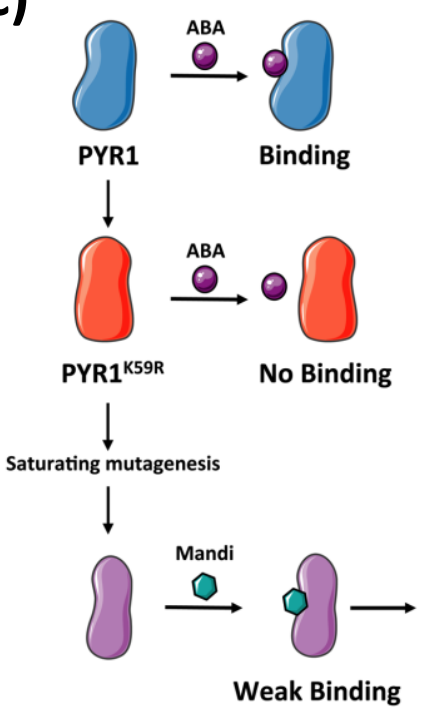

(d)

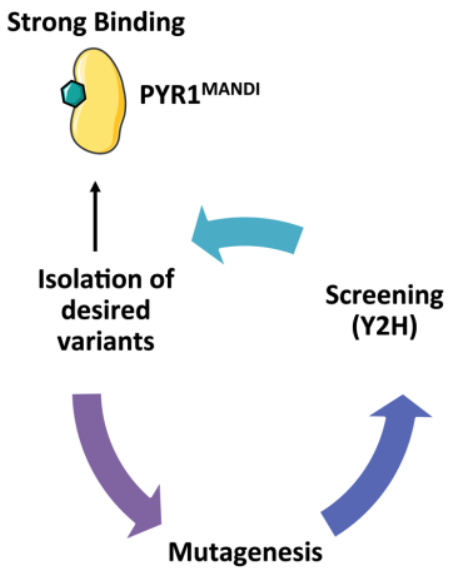

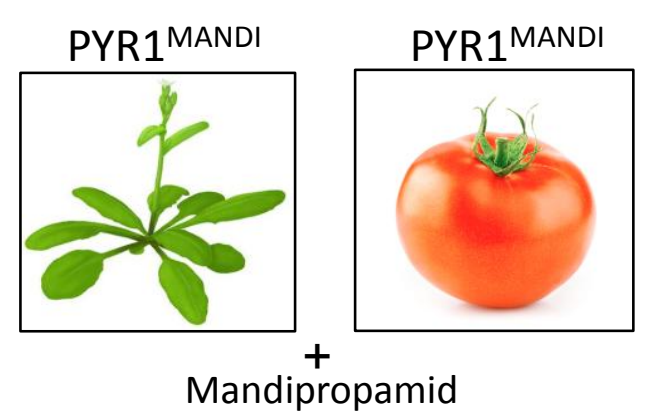

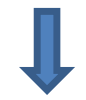

ABA signaling ON

regulation of water loss, support root growth, hydrotropism, accumulation of compatible solutes, synthesis of protective dehydrins/LEAs, ROS detoxification

Figure 1. ABA signaling is controlled in a ligand-dependent manner. (a) Dimeric PYR1, a member of the PYR/PYL/RCAR family of ABA receptors, perceives ABA intracellularly and in its monomeric state forms stable ternary complexes with clade A PP2Cs, thereby inactivating them. It allows the phosphorylation and activation of SnRK2s, which in turn phosphorylate different targets in plant cells to mount the ABA response under stress conditions. (b) Different approaches have been followed to identify small molecules able to act as agonists or antagonists of ABA signaling. The structure of a 3'-alkylsulfanyl-ABA with a 6-C extension (AS6), which acts as ABA-antagonist, is shown. Quinabactin is a sulfonamide ABA-agonist. Mandipropamid is a mandelamide orthogonal agonist able to activate an engineered PYR1 receptor (PYR1MANDI). Images were generated with the LigandScout software tool using 3NEF, 3QN1, 4LA7 and 4WVO Protein Data Bank codes. (c) An agrochemical can be converted into an ABA orthogonal agonist through the use of engineered ABA receptors. First, the K59R mutation abolishes ABA-binding capacity of PYR1. Second, weak binding of agrochemicals can be detected in receptor allele collections where the ABA-binding pocket has been mutagenized. Finally, combinatorial and shuffling mutagenesis generates an engineered PYR1 receptor that shows high affinity for binding of a certain agrochemical. (d) The ABA signaling pathway can be activated in Arabidopsis or tomato plants expressing PYR1 ${ }^{\mathrm{MANDI}}$ through mandipropamid treatment. 